\title{
Pierre Auger Data, Photons, and Top-Down Cosmic Ray Models
}

\author{
Nicolas Busca, ${ }^{1,2,3}$ Dan Hooper, ${ }^{1}$ and Edward W. Kolb ${ }^{1,3}$ \\ ${ }^{1}$ Fermi National Accelerator Laboratory, \\ Particle Astrophysics Center, Batavia, IL 60510-0500 \\ ${ }^{2}$ Kavli Institute of Cosmological Physics, \\ The University of Chicago, Chicago, IL 60637-1433 and \\ ${ }^{3}$ Department of Astronomy \& Astrophysics, \\ The University of Chicago, Chicago, IL 60637-1433
}

(Dated: February 5, 2008)

\begin{abstract}
We consider the ultra-high energy cosmic ray (UHECR) spectrum as measured by the Pierre Auger Observatory. Top-down models for the origin of UHECRs predict an increasing photon component at energies above about $10^{19.7} \mathrm{eV}$. Here we present a simple prescription to compare the Auger data with a prediction assuming a pure proton component or a prediction assuming a changing primary component appropriate for a top-down model. We find that the UHECR spectrum predicted in top-down models is a good fit to the Auger data. Eventually, Auger will measure a composition-independent spectrum and will be capable of either confirming or excluding the quantity of photons predicted in top-down models.
\end{abstract}

PACS numbers: 95.55.Vj;96.50.sb;98.70.Sa FERMILAB-PUB-06-022-A 


\section{INTRODUCTION}

The origin of the highest energy cosmic rays has been a subject of great interest for some time. The particular aspect of Ultra-High Energy Cosmic Ray (UHECR) physics that has raised the greatest degree of interest is the question of whether the cosmic ray spectrum exhibits a feature known as the GZK cutoff [1]. This cutoff is the result of the suppression of the cosmic ray spectrum above a few $10^{19} \mathrm{eV}$ due to protons interacting with the cosmic microwave background. If a GZK suppression is not observed, it would indicate that the sources of these ultra-high energy events would be of local origin, cosmologically speaking (within 10 to $50 \mathrm{Mpc}$ ). However, no nearby astrophysical sources sources capable of accelerating particles to such high energies are known to exist.

Measurements of the UHECR spectrum have not clearly settled the issue of whether a GZK suppression is present. On one hand, the spectrum measured by the AGASA experiment shows no indication of a GZK suppression [2]. In particular, in Ref. [3] it is shown that the number of events with energies above $10^{20} \mathrm{eV}$ expected in a GZK scenario is 3.6, while the number of events observed by AGASA is 11, corresponding to a significance of 3 standard deviations. In contrast, the HiRes experiment appears to have observed the presence of a GZK suppression: Ref. [4] concludes that HiRes data exclude a non-GZK scenario with a significance of 3 to 4 standard deviations. Given this discrepancy, it appears that further data would be required to resolve the question at hand. In particular, the Pierre Auger Observatory (or simply Auger) is currently under construction at its southern site in Argentina. Auger combines the techniques used by AGASA (an air shower ground array) and HiRes (fluorescence detectors) allowing it to make energy spectrum measurements which are less composition and model dependent than either HiRes or AGASA.

Auger's first results were released in 2005, but did not clearly resolve the question of whether a GZK feature is present in the UHECR spectrum. These data was collected with only a fraction of the southern site completed, but yet the total exposure at this point was slightly larger than the total accumulated by AGASA. Auger calibrated their ground array data using their florescence detectors, resulting in a largely composition independent energy measurement. Due to a lack of events, this hybrid calibration was only possible at energies well below the GZK cutoff, however, and if the composition of the UHECR spectrum charges between the calibration energies and higher energies, then the highest energy bins in the published Auger spectrum must be modified.

This effect is particularly pronounced in the case of photon primaries. Auger's ground ar- 
ray measures energy with a parameter known as $\mathrm{S}(1000)$ [5], which is proportional to the water Cherenkov signal in the surface array at a distance of 1000 meters from the shower axis. Due to the lack of muons generated by photon primaries, photon-induced events would produce a smaller $\mathrm{S}(1000)$ than proton-induced events of the same primary energy. Therefore, the actual cosmic ray flux may be considerably larger at the highest energies than reported by Auger if a substantial fraction of the highest energy cosmic rays are photons.

A substantial fraction of the highest energy cosmic rays are expected to be photons in topdown cosmic ray models. In this article, we consider the effect that this will have on the UHECR spectrum observed by Auger. In particular, we show that Auger's data result in a spectrum without the appearance of a GZK cutoff if the photon fraction of UHECRs follows the prediction of topdown models.

\section{TOP-DOWN COSMIC RAY MODELS}

If no GZK cutoff is found to be present in the UHECR spectrum, then either local (within 10 to $50 \mathrm{Mpc}$ ) sources of UHECRs must exist [6], or some kind of exotic physics must be invoked to evade the GZK effect. Among exotic possibilities, proposals have included UHECRs composed of exotic hadrons [7], or strongly-interacting neutrinos [8], or that protons can travel super-GZK distances due to a violation of Lorentz invariance [9]. The solution to the UHECR problem that we focus on in this article is a top-down scenario, in which the highest energy cosmic rays are generated locally (in our galaxy) by the decays of supermassive particles [10] or topological defects [11].

Unlike other proposed scenarios, in top-down models the highest energy cosmic rays are mostly photons. For this reason, it can be misleading to compare the spectrum presented by the Auger collaboration to the predicted spectrum in these models. In Fig. 11 we compare the published Auger data to a spectrum of protons from homogeneous astrophysical sources (left frame) and to the same spectrum plus a top-down component (right frame). We find, somewhat unexpectedly, that the data fits both scenarios reasonably well.

In order to compare the Auger data with the predictions of a top-down model we consider the effect a proton-photon mixed primary composition would have on the Auger energy calibration curve. If at the present energy calibration range most of the primaries are protons, then the energy of a primary photon would be underestimated by a factor of two [5]. Consider a photon fraction 

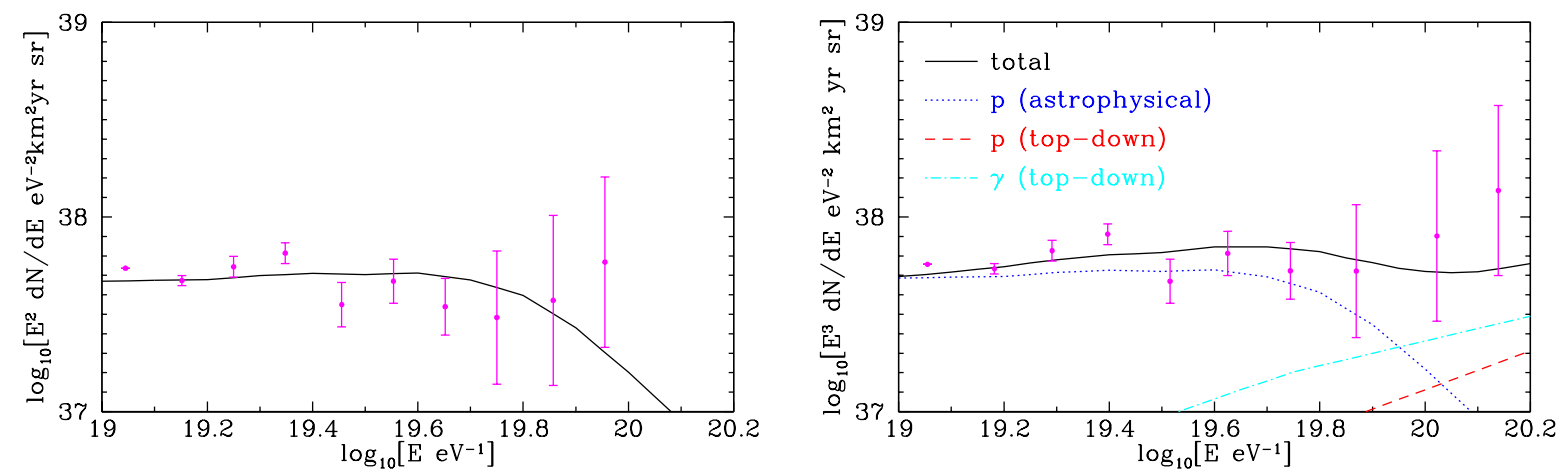

FIG. 1: In the left frame, we plot the data from the Pierre Auger Observatory compared to the spectrum from a conventional astrophysical model of homogeneously distributed sources with an injection spectrum of $d N_{p} / d E_{p} \propto E_{p}^{-2.8}$. In the right frame, we plot the same conventional source spectrum along with the spectrum from the decay of supermassive particles or topological defects of mass $M_{X}=6 \times 10^{21} \mathrm{eV}$. In the right frame, the Auger data has been shifted to account for the photon composition in the top-down spectrum. Both models fit the data quite well.

$f\left(E, P_{0}, M_{X}\right)$, which is a function of the energy $E$, the injection power $P_{0}$, and the mass of the decaying particle $M_{X}$, which is small at the Auger energy calibration range $\left(10^{18} \sim 10^{19.4}\right)$. Above this range a change in the calibration curve would lead to the following shift on the energy:

$$
E_{\text {shift }}=E\left[1-f\left(E_{\text {shift }}, P_{0}, M_{X}\right)+2 f\left(E_{\text {shift }}, P_{0}, M_{X}\right)\right]
$$

where $E_{\text {shift }}$ is the shifted energy. For each bin of mean energy $E$ in the published Auger spectrum, we solved this equation to find the corresponding shifted energy $E_{\text {shift }}$. We finally chose the values of $P_{0}$ and $M_{X}$ that best fit the data.

The photon fraction of the UHECRs in the model shown in the right frame of Fig. 1 is shown in Fig. 2. The spectrum and composition of UHECRs generated in top-down models has been calculated using the publicly available program SHDECAY [15]. We have shown results here for the case of decays to quark-antiquark pairs, and assumed the presence of supersymmetry, although our conclusions are largely insensitive to these choices. 


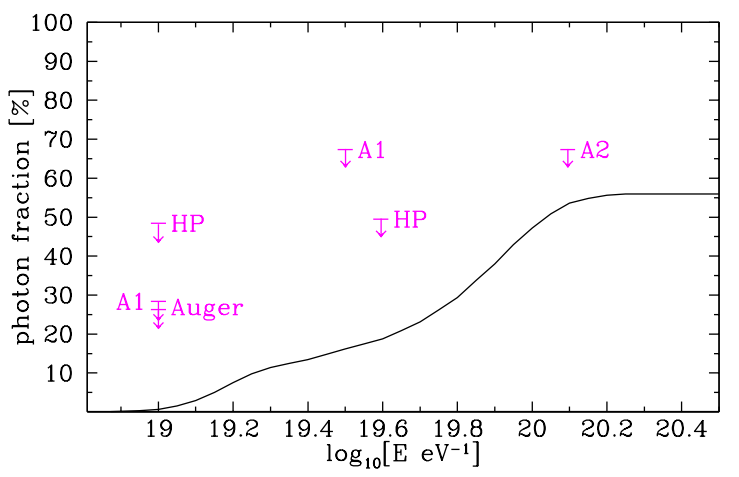

FIG. 2: The fraction of photons in the UHECR spectrum as a function of energy in the top-down model shown in the right frame of Fig. 1] This prediction is clearly below the limits set by the Auger [12], Haverah Park (HP) [13] or AGASA (A1, A2) data [14].

\section{FUTURE PROSPECTS FOR CONFIRMING OR EXCLUDING TOP-DOWN MODELS}

Although we have shown here that present UHECR data (Auger data in particular) is not capable of confirming or excluding top-down models, the prospects for testing such models in the near future are very promising. These prospects come from at least four types of observations: future improvement on the systematic uncertainty on the energy measurement, future UHECR photon fraction measurements, future UHECR anisotropy measurements, and future ultra-high energy neutrino measurements.

Auger will certainly improve its systematic uncertainties in the near future, leading to a better established energy spectrum. Such a spectrum would clearly resolve the issue of the existence of a GZK cutoff and confirm or rule out the hypothesis of an additional high energy component.

As Auger accumulates more data, its hybrid detector will be able to place limits on the photon fraction at increasingly high energies. Currently, the Auger photon limit only constrains the composition above $10^{19} \mathrm{eV}$ to be less than $26 \%$ photons (at the $95 \%$ confidence level) [12]. To test most top-down scenarios, a similar constraint would be needed at a much higher energy, perhaps around $10^{19.7} \mathrm{eV}$. Auger will accumulate an exposure sufficient to accomplish this only after about 5-6 years of operation with a full southern array.

Auger will also be capable of studying the isotropy of the UHECR spectrum to unprecedented levels of precision. If a substantial fraction of the highest energy events are generated in topdown decays within the galactic halo, this can lead to an observable level of anisotropy directed 
toward the center of our galaxy. To identify such an isotropy, several hundred events of the highest energies will be required. It has been estimated that such a signal could be resolved at Auger South after 3 years of operation with a full array [16]. Current Auger data only constrains anisotropies at 0.8-3.2 EeV [17], well below the range effected by top-down models.

In addition to generating ultra-high energy photons and protons, top-down decays produce a large number of neutrinos. Such a flux of neutrinos is expected to result in of the order of one event in the first ANITA flight, scheduled for later in 2006. Other experiments such as IceCube and Auger are expected to reach the sensitivity needed to detect top-down neutrinos as well [18]. Such neutrinos will be diffuse and difficult to identify as being of top-down origin, however. The rates anticipated from ultra-high energy proton interactions with the cosmic microwave background (the cosmogenic neutrinos flux) are similar to those for top-down models, and are virtually impossible to distinguish from each other. The lack of such events, on the other hand, would be a fairly compelling piece of evidence against top-down models, and may imply a substantial component of heavy nuclei in the UHECR spectrum [19].

Among these four classes of observation, top-down models should testable within the next several years, and are likely to be either experimentally excluded or confirmed.

\section{SUMMARY AND CONCLUSIONS}

The calibration of the Pierre Auger Observatory has been made using a hybrid technique at sub-GZK energies. This fact leads to a large systematic uncertainty at the highest energies of the order of about $40 \%$. A change in composition above Auger calibration energies might lead to a systematic shift in their higher energy events, even to the full extent of this uncertainty. This is particularly true in top-down cosmic ray models, in which the highest energy cosmic rays are generated in the decays of super-massive particles or topological defects. In these models many of the highest energy cosmic rays are photons, which have their energies underestimated by about $50 \%$ at Auger.

In this article, we calculated the expected shift on the Auger spectrum assuming the photon content of a typical top-down model. This shift is consistent with the quoted experimental systematic uncertainty and is toward higher energies. We find that the resulting spectrum agrees quite well to the top-down prediction. We also showed that the spectrum is consistent with a pure extragalactic proton hypothesis where no shift is needed. 
As the Pierre Auger Observatory accumulates more data, its ability to calibrate in a composition independent fashion will be applied at increasingly higher energies. At least 5-6 years of exposure with a full southern array will be required to reach the energy at which photons begin to dominate the UHECR spectrum in top-down models, however. Anisotropy measurements by Auger may also be able to test top-down models after a few years of observation, and upcoming ultra-high energy neutrino measurements will be relevant to top-down models as well.

Acknowledgments: We would like to thank Andrew Taylor for providing the conventional proton spectrum used in Fig. 1. We would also like to thank Aaron Chou for interesting discussions. This work has been supported by the US Department of Energy and by NASA grant NAG5-10842.

[1] K. Greisen, Phys. Rev. Lett. 16, 748 (1966); G. T. Zatsepin and V. A. Kuzmin, JETP Lett. 4, 78 (1966) [Pisma Zh. Eksp. Teor. Fiz. 4, 114 (1966)].

[2] M. Takeda et al., Astropart. Phys. 19447 (2003)

[3] D. De Marco, P. Blasi, A.V. Olinto, arXiv:astro-ph/0507324

[4] D.R. Bergman [HiRes Collaboration], arXiv:astro-ph:/0507484

[5] P. Sommers [Pierre Auger Collaboration], arXiv:astro-ph/0507150

[6] For example, see: C. Isola, M. Lemoine and G. Sigl, Phys. Rev. D 65, 023004 (2002) arXiv:astro-ph/0104289;; P. Blasi and A. V. Olinto, Phys. Rev. D 59, 023001 (1999) [arXiv:astro-ph/9806264].

[7] I. F. M. Albuquerque, G. R. Farrar and E. W. Kolb, Phys. Rev. D 59, 015021 (1999) arXiv:hep-ph/9805288.

[8] P. Jain, D. W. McKay, S. Panda and J. P. Ralston, Phys. Lett. B 484, 267 (2000) arXiv:hep-ph/0001031; G. Domokos, S. Kovesi-Domokos and P. T. Mikulski, arXiv:hep-ph/0006328

[9] F.W. Stecker and S.T. Scully Astropart. Phys. 23,203 (2005)

[10] V. Berezinsky, M. Kachelriess and A. Vilenkin, Phys. Rev. Lett. 79, 4302 (1997) arXiv:astro-ph/9708217;; M. Birkel and S. Sarkar, Astropart. Phys. 9, 297 (1998) arXiv:hep-ph/9804285; D. J. H. Chung, E. W. Kolb and A. Riotto, Phys. Rev. D 59, 023501 (1999) arXiv:hep-ph/9802238 ; P. Blasi, R. Dick and E. W. Kolb, Astropart. Phys. 18, 57 (2002) arXiv:astro-ph/0105232; D. J. H. Chung, P. Crotty, E. W. Kolb and A. Riotto, Phys. Rev. D 64, 
043503 (2001) arXiv:hep-ph/0104100]; S. Sarkar and R. Toldra, Nucl. Phys. B 621, 495 (2002) [arXiv:hep-ph/0108098].

[11] P. Bhattacharjee and G. Sigl, Phys. Rev. D 51, 4079 (1995) [arXiv:astro-ph/9412053]; G. Sigl, Space Sci. Rev. 75, 375 (1996) [arXiv:astro-ph/9503014]; V. Berezinsky and A. Vilenkin, Phys. Rev. Lett. 79, 5202 (1997) [arXiv:astro-ph/9704257].

[12] M. Risse [Pierre Auger Collaboration], arXiv:astro-ph/0507402

[13] M. Ave et al., Phys.Rev.Lett. 852244 (2000) M. Ave et al., Phys. Rev. D 65063007 (2002)

[14] K. Shinozaki et al. Astrophys. Journal. 571 L117 (2002) M. Risse et al. arXiv:astro-ph/0502418

[15] C. Barbot, Comput. Phys. Commun. 157, 63 (2004) [arXiv:hep-ph/0306303]; C. Barbot and M. Drees, Astropart. Phys. 20, 5 (2003) [arXiv:hep-ph/0211406]; C. Barbot and M. Drees, Phys. Lett. B 533, 107 (2002) arXiv:hep-ph/0202072].

[16] N. W. Evans, F. Ferrer and S. Sarkar, Astropart. Phys. 17, 319 (2002) [arXiv:astro-ph/0103085].

[17] A. Letessier-Selvon [Pierre Auger Collaboration], arXiv:astro-ph/0507331.

[18] C. Barbot, M. Drees, F. Halzen and D. Hooper, Phys. Lett. B 555, 22 (2003) [arXiv:hep-ph/0205230].

[19] D. Hooper, A. Taylor and S. Sarkar, Astropart. Phys. 23, 11 (2005) arXiv:astro-ph/0407618; M. Ave, N. Busca, A. V. Olinto, A. A. Watson and T. Yamamoto, Astropart. Phys. 23, 19 (2005) arXiv:astro-ph/0409316. 